\title{
Research on Laser Terminal Guidance Cannonballs Attack Characteristics at the End
}

\author{
Ming Jiang, Yun Li, Yu-Wen Liu, Yong Li \\ New Star Research Institute of Applied Technology, HeFei, 230031, China \\ E-mail: 389555317@qq.com
}

\begin{abstract}
In the paper, we did some research on the terminal guidance cannonballs' capture field, attack area, the effective attack area, and the influence factors. The study was based on computer, through 6D ballistic model, Matlab program, and computer calculation. The boundary value of attack area along with positive shoots direction is bigger than negative about $20 \mathrm{~m}$ to $80 \mathrm{~m}$. The conclusion is that, as to have the more properly effective attack area, the start control height of terminal guidance is in commonly $1200 \mathrm{~m}$ advisable, the initial trajectory angle choice $-26^{\circ}$ is or so for proper, and the terminal part initial rate of the shell can't be too small and generally selected by around $230 \mathrm{~m} / \mathrm{s}$.
\end{abstract}

Keywords-terminal guidance; ballistic; attack characteristics; simulation

Precise hit is going to be the main type of war. The terminal guidance shell is a shell which can be projected by the conventional cannon, but precisely guided during the terminal part of the trajectory, and there are many countries in the world doing research on this shell. The shell called "Krasnopol" produced by Russia is a kind of terminal homing shell. This kind of high-tech shells, made the cannon, which is indirect sighting antipersonnel weapon, be able to precisely strike point target(armored target) from a long distance. However, we should know clearly about these cannonballs' attack characteristics at the end, for example, the capture field, the attack area, the effective attack area, etc. Further more, some influence factors may be affected the attack characteristics, so what were them, and how they did. In the paper, we did some research on the attack characteristics at the end, including the capture field, the attack area, the effective attack, and the influence factors. The study was based on computer, through ballistic model, program, and calculation. The research results may have certain significance on the improvement of this type of laser terminal homing cannonballs.

\section{THE CHARACTERISTICS OF CAPTURE FIELD}

The homing head of shell began working before the end guidance part of trajectory. After accepting laser signal reflected back by target, it succeeded in catching target. Usually, the cannonball just can catch the target distributed in the certain district, and this district is usually called “capture field”.
At the catching beginning, the direction of homing head's optical axis and the shell's primary axis are superposed. The relation and the sketch map of capture field are shown such as the figure 1 .

In the picture, $H$ is flight height, $\varphi$ is attitude angle. At the beginning, $\varphi$ is same with trajectory tilt angle $\theta . \psi_{r}$ is the field angle, which limits the flying space into a cone. When the cone intersects the horizontal plane, a cone piece line is got. In the meantime, $D_{r}$ is the effective distance of the homing head, which limits the flying space into a sphere. The sphere intersecting the horizontal plane gets a circle line. The area between the cone piece line and the circle line is the capture field of the homing head. It is briefly called capture field ${ }^{[1 \sim 2]}$.

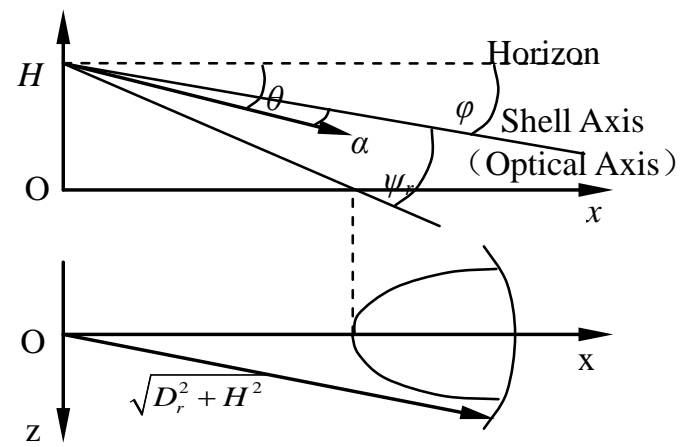

Figure 1. Sketch map of capture field.

When target is inside the area, the shells can accept laser signal reflected back from the target, then, control instruction can be formed. Whereas, if outside, the bullet could not succeed in catching a target.

The size of capture field is influenced by the factors: flight height $H$, attitude angle $\varphi$, field angle $\psi_{r}$, and effective distance $D_{r}$. Thus, the cone piece line, which build the boundary of capture field, has a different property when the attitude angle and optical angle change relatively. 


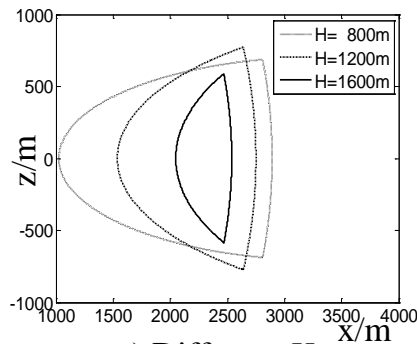

a) Different $H$

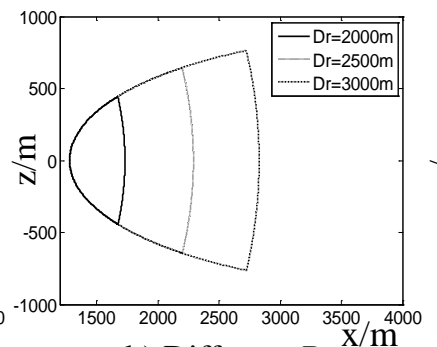

b) Different $D_{r}$

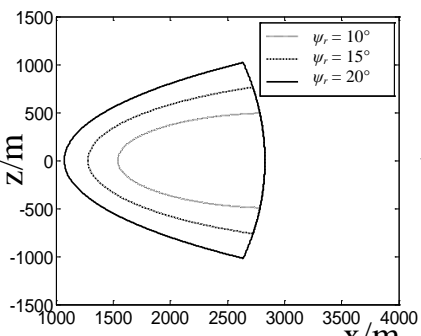

c) Different $\psi_{r}$

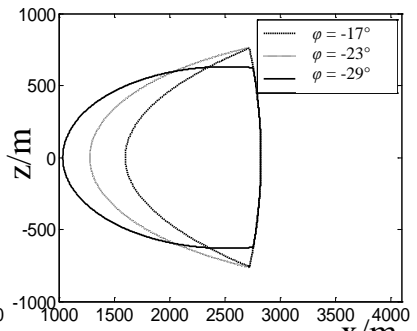

d) Different $\varphi$

Figure 2. The capture field with different condition

When $\varphi<\psi_{r}$, the cone piece line is the single side of hyperbola; When $\varphi=\psi_{r}$, the cone piece line is parabola; When $\varphi>\psi_{r}$, the line is oval line.The circumstance for terminal homing shells is generally the third.

When trajectory simulation was done, it mainly computed above factor variety's influence on the capture field. The result is shown as figure 2 .

The figure 2a) shows the capture field when $\varphi=-23^{\circ}$, $\psi_{r}=15^{\circ}, D_{r}=3000 \mathrm{~m}, H$ changing. The figure $2 \mathrm{~b}$ ) shows the capture field when $\varphi=-23^{\circ}, \psi_{r}=15^{\circ}, H=1000 \mathrm{~m}, D_{r}$ changing. The figure 2c) shows the capture field when $\varphi=$ $-23^{\circ}, D_{r}=3000 \mathrm{~m}, H=1000 \mathrm{~m}, \psi_{r}$ changing. The figure $2 \mathrm{~d}$ ) shows the capture field when $\psi_{r}=15^{\circ}, D_{r}=3000 \mathrm{~m}, H=$ $1000 \mathrm{~m}, \varphi$ changing.

It could be known by the diagram that the capture field diminishing with height growing diminishing, growing with effective distance increasing, growing with optical angle becoming bigger, diminishing with attitude angle growing.

\section{ATTACK AREA CHARACTERISTIC}

\section{A. The Establishment of the Attack Area}

Terminal guidance cannonballs are used to attack target precisely, especially for the armor coping. However, the area of armor coping is too small. If impact point and target point have deviation above $1.0 \mathrm{~m}$, the attacking was failing. So, the error less than $1.0 \mathrm{~m}$, is the successful attack standard. All the impact point conforming it constituted the attack area.

Fist of all, the calculation model is the $6 \mathrm{D}$ ballistic model established in document [3]. The difference is that when this paper was using it, modification was given to the wind, which was regarded as zero, no wind speed, no wind direction.

Secondly, the air dynamic coefficient is the test data from wind tunnel experiment.

Thirdly, the calculation platform is Matlab, in which the calculating program was written and operating.

In order to attain the attack area, the paper computed the model in different artillery range and different launching direction as different targets.

The computing treatment is alternately changing the value of target coordinate, making sure the distance is $\Delta x$ between points on the artillery range, and the distance is $\Delta z$ on the launching direction.

Then, there formed many meshes in the horizontal plane. At each point of mesh, carried on a ballistic calculation, and carried on accuracy judgment, thus got the attack area, such as figure 3 . During the attack area calculation in the paper, took $\Delta x=100 \mathrm{~m}, \Delta z=20 \mathrm{~m}$.

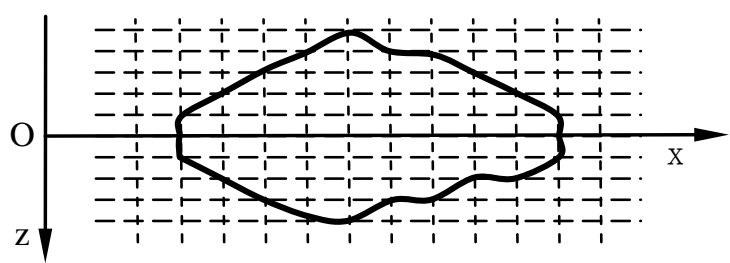

Figure 3. $\quad$ Confirm attack area

\section{B. Factors Influencing the Attack Area}

The main factors influencing the attack area of terminal guidance shell are the initial height, the initial trajectory angle and the initial rate of the terminal part of the trajectory.

1) Influence of initial height to attack area: The attack area of initial trajectory angle $\theta=-26^{\circ}$, initial rate $v$ $=230 \mathrm{~m} / \mathrm{s}$ and different initial heights $\mathrm{H}$ was shown in figure 4a. The figure shows that with the increase of height, the near boundary and the farther boundary of the attack area increase along the direction of range; the both sides of the border increase along the direction of shoots; the size of attack area is increased. Each additional $200 \mathrm{~m}$ height, the near boundary moves forward about $100 \mathrm{~m}$, the farther boundary moves forward about $400 \mathrm{~m}$ to $1200 \mathrm{~m}$, the both sides of border increase about $100 \mathrm{~m}$. 


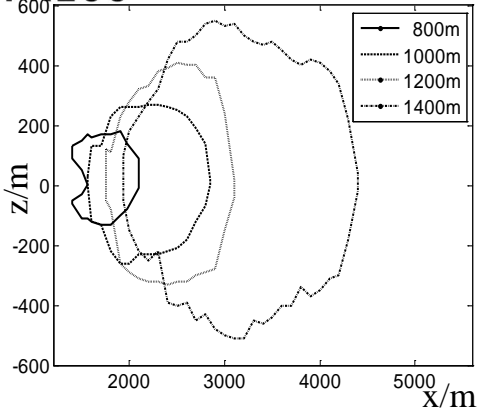

a) Different Initial Height

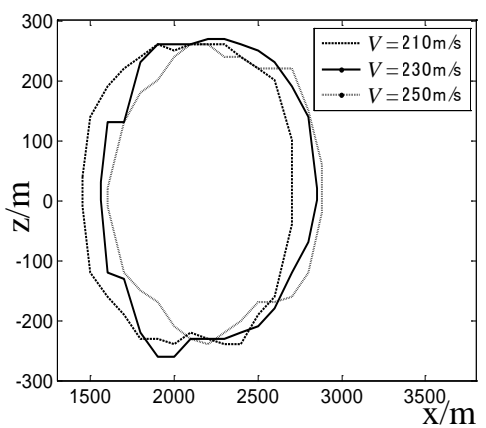

b) Different Initial Rate

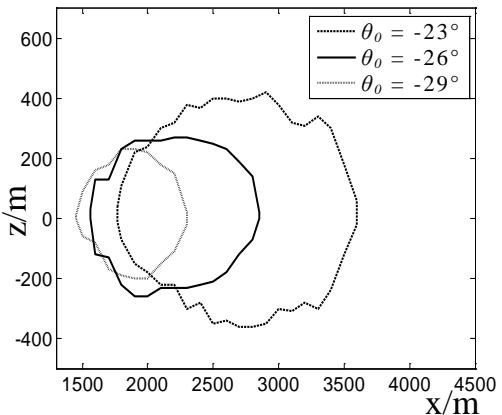

c) Different Initial Trajectory Angle

Figure 4.

Attack Area with Different Initial Conditions

2) Influence of initial rate to attack area: The attack area of initial trajectory angle $\theta=-26^{\circ}$, initial height $\mathrm{H}=1000 \mathrm{~m}$ and different initial rate $\mathrm{v}$ was shown in figure $4 \mathrm{~b}$. The figure shows that with the increase of rate, the near boundary and the farther boundary of the attack area increase along the direction of range, but the increasing distance is not big and no more than $100 \mathrm{~m}$; the both sides of border are essentially unchanged; the size of attack area also has small change.

3) Influence of initial trajectory angle to attack area: The attack area of initial rate $v=230 \mathrm{~m} / \mathrm{s}$, initial height $\mathrm{H}=1000 \mathrm{~m}$ and different initial trajectory angle $\theta$ was shown in figure 4c. The figure shows that with the increase of trajectory angle, the near boundary and the farther boundary of the attack area increase along the direction of range; the both sides of border increase along the direction of shoots; the size of attack area is increased.

\section{The Characteristics of Attack Area}

Synthesizes the calculation of attack area can discover that, the boundary value of attack area along with positive shoots direction is bigger than negative about $20 \mathrm{~m}$ to $80 \mathrm{~m}$. From figure 4 can be seen that the shape of attack area is asymmetric distribution about $\mathrm{z}=0$. It also suggests that the flight ability along with positive shoots direction of terminal guidance shell is better than that of negative.

\section{EFFECTIVE ATTACK AREA CHARACTERISTIC}

\section{A. The Effective Attack Area}

The effective attack area is the intersection area of capture field and attack area. The capture field of terminal guidance shell and the attack area was analyzed above. If the capture field is the visible area of terminal guidance shell, the attack area is the pinpoint shooting area of terminal guidance shell, and then the effective attack area is both the visible area and the pinpoint shooting area of terminal guidance shell. The effective area is the real tactics against effect area of terminal guidance shell.

\section{B. Factors Influence the Effective Attack Area}

The main factors influencing the effective attack area are the initial height, the initial trajectory angle and the initial rate of the shell's terminal trajectory. Figure 5, figure 6 and figure 7 had given the effective attack area diagram of different initial height, initial trajectory angle and initial rate respectively.

1) Influence of initial height to effective attack area: From I and II can be known that the initial height influence the size of capture field as well as the size of attack area. Along with the increase of initial height $\mathrm{H}$, the capture field decreased but the attack area increased, it can not in brief say that the effective attack area becomes big or diminishes like this. Figure 5 was the effective attack area diagram of initial rate $v=230 \mathrm{~m} / \mathrm{s}$, initial trajectory angle $\theta=-26^{\circ}$ and different initial height $H$. It can be discovered from the figure that the effective attack area with height variation is not obvious, but the ratio of the effective attack area and attack area changes obviously. The area ratio of the effective attack area and attack area was significantly reduced as the height increasing. It's disadvantageous for efficient use of terminal guidance shell's terminal attack ability when $H$ was larger. Increase the ratio must increase the capture field. The back curve of capture field was taper cutting line which was limited by field of view. Field of view was $15^{\circ}$ in simulating calculation, at this time, as long as the seeker's effective distance was large enough, attack area was within the limited scope of the cutting line. Because the front curve of capture field is the arc limited by seeker's effective distance, enlarge the capture field need to enlarge the radius of arc, that is enlarging the effective distance of seeker. Thereby, enlarge the ratio must enlarge the effective distance of seeker, but in the condition of the effective distance of seeker is settled, enlarge the ratio can only reduce initial height. Hence, both in order to make the effective attack area is biggest, and in order to make the ratio of effective attack area and attack area is bigger. Give full play to the attack ability of terminal guidance shell, the start control height of terminal guidance is in commonly $1200 \mathrm{~m}$ advisable. 


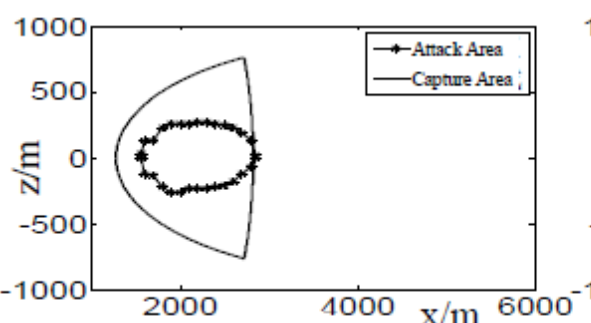

a) $H=1000 \mathrm{~m}$

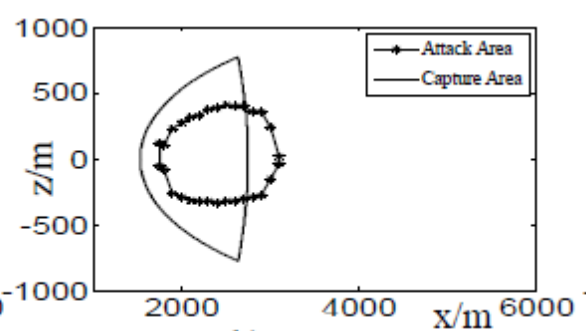

b) $H=1200 \mathrm{~m}$

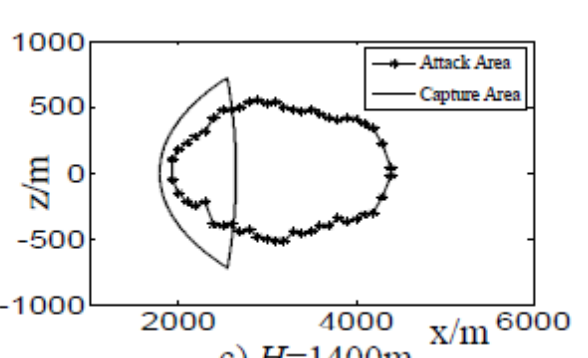

c) $H=1400 \mathrm{~m}$

Figure 5. The effective attack area with different initial height

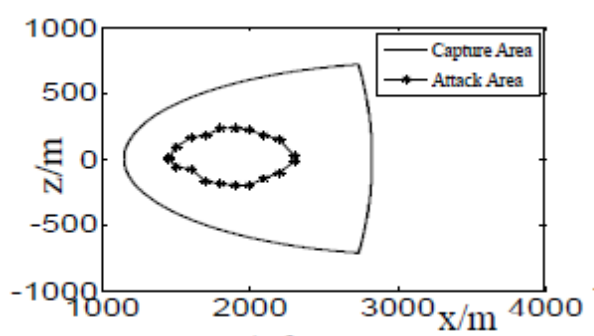

a) $\theta_{0}=-29^{\circ}$

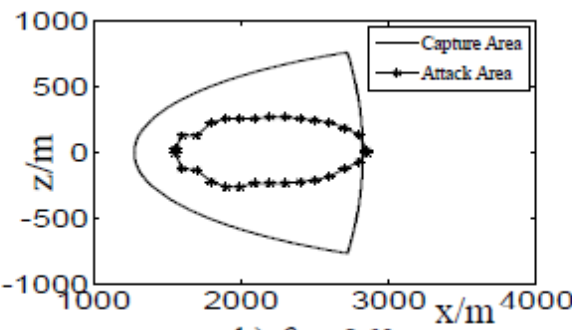

b) $\theta_{0}=-26^{\circ}$

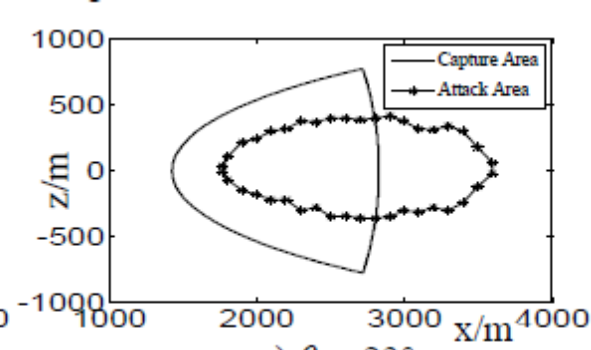

c) $\theta_{0}=-23^{\circ}$

Figure 6. The effective attack area with different initial trajectory angle

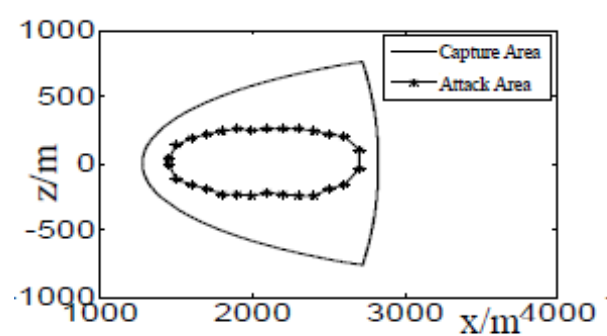

a) $V_{m}=210 \mathrm{~m} / \mathrm{s}$

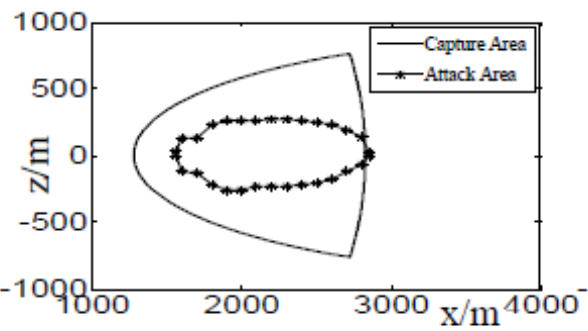

b) $V_{m}=230 \mathrm{~m} / \mathrm{s}$

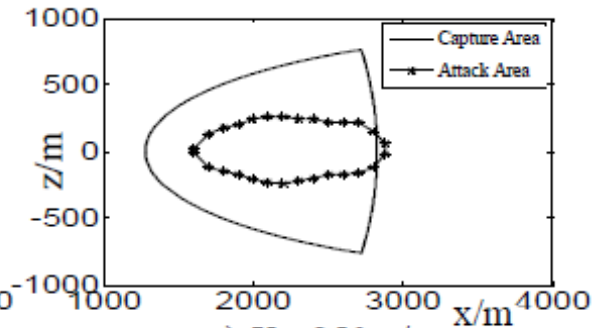

c) $V_{m}=250 \mathrm{~m} / \mathrm{s}$

Figure 7. The effective attack area with different initial rate

2) Influence of initial trajectory angle to the effective attack area: It can be known above ( I and II), the initial trajectory angle influence the size of attack area, the initial attitude angle influence the size of capture field. Because the terminal guidance shell gliding flight almost a linear in inertial navigation, attack angle keeps at around $3^{\circ}$. So you can think that the trajectory angle is always smaller $3^{\circ}$ than the attitude angle at the starting point of terminal guidance. In this case, the change of trajectory angle will influence the size of capture field, and its characteristics are same as that of the attitude angle. Along with the increase of trajectory angle, attack area enlarges and capture field diminishes, it can not in brief say that the effective attack area becomes big or diminishes either. Figure 6 was the effective attack area diagram of initial rate $v=230 \mathrm{~m} / \mathrm{s}$, initial height $\mathrm{H}=1000 \mathrm{~m}$ and different initial trajectory angle $\theta$. The figure showed that the ratio of the effective attack area and attack area had the tendency of decrease along with the increase of initial trajectory angle. That was disadvantageous for effective use of terminal guidance shell's terminal attack ability when $\theta$ was bigger. Want enlarge the ratio, also need to enlarge the effective distance of seeker. But in the case of seeker's effective distance is settled, enlarge the ratio can only decrease the initial trajectory angle. Accordingly, both in order to make the effective attack area is biggest, and in order to fully play the attack ability of terminal guidance shell, the initial trajectory angle 
choice $-26^{\circ}$ is or so for proper.

3) Influence of initial rate to the effective attack area: It can be known from the analysis above ( I and II ), initial rate influence the size of attack area only, but have not effect on that of capture field. Figure 7 is the diagram of effective attack area when the initial trajectory angle $\theta=-26^{\circ}$, the initial height $H=1000 \mathrm{~m}$ and initial rate $v$ is different. From the figure can be seen that, the capture field is not change under this circumstance, the attack area is basically in the scope of capture field, and i.e. the ratio of the effective attack area and attack area is 1 . But with the increase of initial rate, the attack area has the trend of beyond the limit scope of capture area's front arc. Just it's not obvious with the above two cases. This explains the initial rate's influence to the effective attack area is not big. But for the sake of better target, the shell must have a certain speed when it hit the target. This requires that the terminal part initial rate of the shell can't be too small and generally selected by around $230 \mathrm{~m} / \mathrm{s}$.

\section{CONCLUSION}

The effective attack area is both the visible area and the pinpoint shooting area of terminal guidance shell, especially the real tactics against effect area. The result of the paper is that, the capture field diminishing with height growing diminishing, growing with effective distance increasing, growing with optical angle becoming bigger, diminishing with attitude angle growing, the boundary value of attack area along with positive shoots direction is bigger than negative about $20 \mathrm{~m}$ to $80 \mathrm{~m}$. The conclusion is that, as to have the more properly effective attack area, the start control height of terminal guidance is in commonly $1200 \mathrm{~m}$ advisable, the initial trajectory angle choice $-26^{\circ}$ is or so for proper, and the terminal part initial rate of the shell can't be too small and generally selected by around $230 \mathrm{~m} / \mathrm{s}$.

\section{REFERENCES}

[1] Study on the Minimal Attack Area of Terminal Homing Shells [D]. Li Li. Rocket Missile Branch Symposium China Ordnance Society. 1998

[2] Research on the simulation of the Terminal Homing Shells' Attack Area [M]. Guoying Li. Ballistic Journal. 1997(1):67 72.

[3] Trajectory Model of Terminal Guided Projectile under Wind Condition [M]. Qinglan Zhao. Journal of Engineering Design. 2008,15(5):373 377. 